\title{
Wiggling Structures Along the NGC 1333 IRAS 2A Outflow
}

\section{Cheng-Hung Tsai ${ }^{1}$, Huei-Ru Chen ${ }^{1,2}$, Chin-Fei Lee ${ }^{2}$, Naomi Hirano ${ }^{2}$, and Hsien Shang ${ }^{2}$}

${ }^{1}$ Institute of Astronomy and Department of Physics, National Tsing Hua University, Hsinchu, Taiwan

Email: arthur770410@gmail.com

${ }^{2}$ Institute of Astronomy and Astrophysics, Academia Sinica, P.O. Box 23-141, Taipei 106, Taiwan

\begin{abstract}
Wiggling structures in a bipolar outflow may be attributed to orbital motion of a binary system or precession of an accretion disk perturbed by a companion. The shocked knots along the outflow axis display a morphology with either mirror symmetry due to the orbital motion or point symmetry resulted from disk precession. Using the Submillimeter Array (SMA), our $\mathrm{CO}$ (2-1) and $\mathrm{SiO}$ (5-4) observations show wiggling structures in the collimated bipolar outflow driven by the NGC 1333 IRAS 2 A Class 0 protostar $(d \sim 200 \mathrm{pc})$. By fitting the peak positions of emission knots, we can examine the lateral displacement of the molecular jet to constrain parameters of the unresolved binary system, such as the binary separation and total binary mass. With an angular resolution of $\sim 3^{\prime \prime}$, we have determined the knot positions in $\mathrm{SiO}(5-4)$ (Fig. 1) and $\mathrm{CO}(2-1)$. As a first attempt, we consider the scenario of orbital motion in a binary system and estimate a total binary mass of $\sim 1 M_{\odot}$ and a binary separation of roughly $\sim 20 \mathrm{AU}$, corresponding to $\sim 0.1^{\prime \prime}$. Such a small separation makes it challenging to resolve this hypothesized proto-binary system, which is thought to be responsible for the large-scale quadrupolar outflow nearly perpendicular with each other in CO (1-0).
\end{abstract}

Keywords. ISM: individual (IRAS 2A) — ISM: jets and outflows

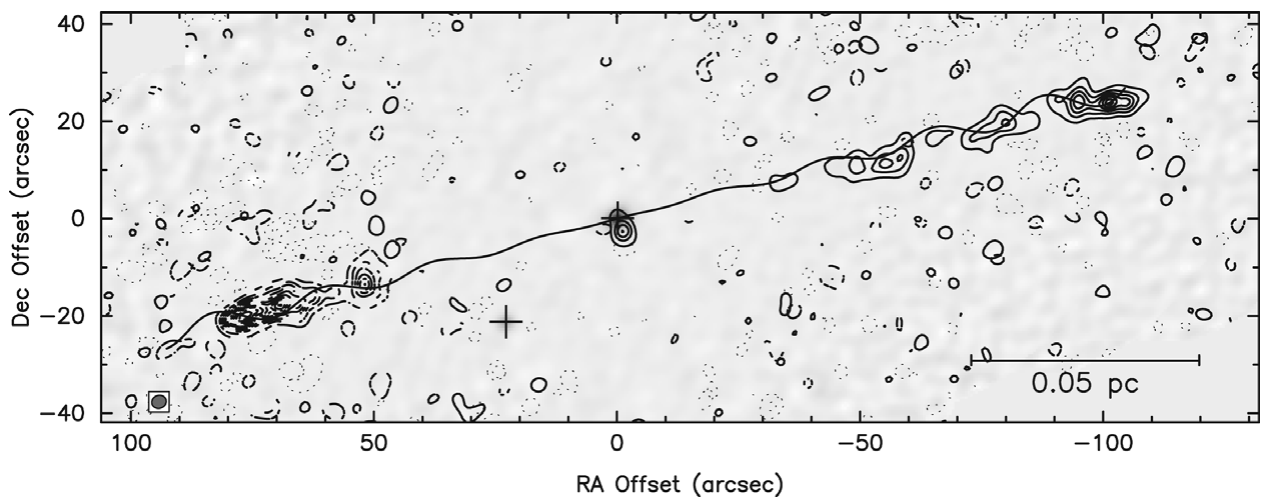

Figure 1. SMA integrated maps of the blue- (thick contours) and red-shifted (thick dash contours) components of the $\mathrm{SiO}(5-4)$ emission overlaid with the $1.3 \mathrm{~mm}$ continuum map (gray scales). Black crosses mark the positions of IRAS $2 \mathrm{~A}$ (center) and IRAS $2 \mathrm{~B}$. The black curve is our best fit result. 